\title{
Stochastic model predictive control for optimal energy management of district heating power plants
}

DOI:

10.1109/CDC.2016.7798367

Document Version

Accepted author manuscript

Link to publication record in Manchester Research Explorer

\section{Citation for published version (APA):}

Verrilli, F., Parisio, A., \& Glielmo, L. (2016). Stochastic model predictive control for optimal energy management of district heating power plants. In 2016 IEEE 55th Conference on Decision and Control (CDC) (pp. 807-812). (IEEE Conference on Decision and Control). IEEE. https://doi.org/10.1109/CDC.2016.7798367

\section{Published in:}

2016 IEEE 55th Conference on Decision and Control (CDC)

\section{Citing this paper}

Please note that where the full-text provided on Manchester Research Explorer is the Author Accepted Manuscript or Proof version this may differ from the final Published version. If citing, it is advised that you check and use the publisher's definitive version.

\section{General rights}

Copyright and moral rights for the publications made accessible in the Research Explorer are retained by the authors and/or other copyright owners and it is a condition of accessing publications that users recognise and abide by the legal requirements associated with these rights.

\section{Takedown policy}

If you believe that this document breaches copyright please refer to the University of Manchester's Takedown Procedures [http://man.ac.uk/04Y6Bo] or contact uml.scholarlycommunications@manchester.ac.uk providing relevant details, so we can investigate your claim.

\section{OPEN ACCESS}




\title{
Stochastic Model Predictive Control for Optimal Energy Management of District Heating Power Plants
}

\author{
Francesca Verrilli ${ }^{1}$, Alessandra Parisio $^{2}$, Luigi Glielmo ${ }^{1}$
}

\begin{abstract}
In this paper a control strategy for the optimal energy management of a district heating power plant is proposed using a stochastic formulation. The main goal of the control strategy is to reduce the production and maintenance costs by optimally managing the boilers, the thermal energy storage and the flexible loads while satisfying a time-varying request and operation constraints. The optimization model includes a detailed modeling of boilers operating constraints, energy thermal energy exchange and the operating modes of the power plant layout. Furthermore, the uncertainty in power demand and renewable power output, as well as in weather conditions, is handled by formulating a two-stage stochastic problem and incorporating it into a model predictive control framework. A simulation evaluation based on the real data and the layout of a Finnish power plant is conducted to assess the performance of our proposed framework. The cost analysis shows the advantages of using the predictive control strategy.
\end{abstract}

\section{INTRODUCTION}

Over the past decades, district heating network (DHN) has gained increased importance in countries with a demand for building heating, such as the Nordic countries. This is due to the centralized heat generation, which increases the possibility of controlling generation and reducing generation costs. In particular, an energy management framework coordinating in an optimal way flexible loads with Combined Heat and Power (CHP) unit, thermal energy storage and Renewable Energy Sources (RES) (e.g. solar thermal power) is then known to be highly beneficial under operational and environmental perspectives [1], [2]. However, this coordinated management can introduce further challenges, particularly due to several sources of uncertainty, e.g., RES power output, energy demand. In this paper, a systematic stochastic framework including all the aforementioned features is designed and evaluated based on the real data and layout of a Finnish power plant. In addition, a feedback mechanism is incorporated through the receding horizon philosophy to better compensate for uncertainty effect.

Several mixed integer linear programming (MILP) models for determining the optimal capacity and operation of CHP plants coupled with district heating and cooling networks to meet the demands of local users have been proposed in the literature [3]-[7], most of which include solar plant and an energy storage device. The results show how an optimized cogeneration coupled with renewables and district heating system allows to reduce significantly both the energy supply

\footnotetext{
${ }^{1}$ Dipartimento di Ingegneria, Università degli Studi del Sannio, Benevento (Italy), \{fverrilli, glielmo\}eunisannio.it

${ }^{2}$ School of Electrical and Electronic Engineering, The University of Manchester, Manchester, United Kingdom alessandra.parisio@manchester.ac.uk
}

cost and the primary energy consumption and to maximize the economic and energy saving benefits of these systems.

Model predictive control (MPC) has drawn increasing attention in the power system community due to the ability of easily integrating predictions, system constraints and a feedback mechanism into the decision making process, which is attractive for systems greatly dependent on demand and renewable energy generation forecasts and uncertainty MPC-based energy management systems has been proposed for several energy systems, such as microgrids (e.g. [8], [9]). Further, for properly handling the various uncertainty sources in energy systems, stochastic programming is considered a promising approach (e.g., [10]-[12]).

Planning frameworks, integrating CHPs and thermal storage techniques, have been proposed in the literature taking the price and demand uncertainties into account [13], [14]. In [15] an optimization model with heat storage is proposed to minimize the production cost. The authors attempt to account for price and demand uncertainty through a twostage process: first the operative plan is optimized over multiple days, then only the first day plan is implemented based on 'actual' values, which however are historical data. The uncertainty is then not incorporated into the planning strategy.

In [16] a model for optimal and dynamic control of CHP-thermal storage in the presence of uncertain market prices is developed. The proposed model is formulated as a stochastic control problem and numerically solved through Least Squares Monte Carlo regression analysis. It is shown how thermal storage can significantly increase CHP flexibility to respond to real time market signals. In [17] a two-stage stochastic MILP model is designed to determine the optimal number and size of CHP system components. The authors in [18] propose a two-stage stochastic programming framework to optimize the use of a thermal energy storage in the form of hot water storage and/or storage in building material. Three outside temperature scenarios and ten price scenarios are combined into thirty scenarios; then, occupied/non-occupied scenarios are randomly assigned.

However, it is notable that, in the current literature, a unified optimization model including modeling of critical, curtailable and shiftable loads, interactions among DERs, renewables and energy storage resources, and influence of uncertain factors, is still to be designed. In addition, the equipment and flexible loads are often modeled through a simplified approach, neglecting different demand side policies and equipment efficiencies and behavior under partial load. 
Extending our previous results, we propose a stochastic control strategy for optimal energy management of a district heating power plant. Special attention is paid also to the modeling of thermal losses and thermal energy storage dynamics [19], [20]. The formulated optimization model includes all the details mentioned above, along with users' preferences and comfort. Further, it is incorporated into a stochastic MPC framework to account for uncertainty in renewables power output and energy demand and take advantage of a feedback mechanism, without suffering of the curse of dimensionality (as dynamic programming approaches). A Sample Average Approximation (SAA) approach is adopted for deriving a minimum number of scenarios in order to obtain a feasible solution of the original stochastic optimization problem [21][23].

Our high-level control strategy computes power and temperature setpoints to the boilers, the scheduling of the thermal energy storage and the flexible loads. A SCADA system on the low-level control architecture is in charge of selecting the appropriate mass flow to guarantee the energy balance equation.

The rest of the paper is organized as follows. In section II, the mathematical formulation of both system dynamics and constraints are provided. The formulation of the overall stochastic MPC problem is shown in section III. In section IV, we test our strategy, in simulation, against the model of an actual district heating power plant located in Finland, providing simulation results and an analysis of the saving costs with respect to the historical data.

\section{A. Nomenclature}

In tables I, II and III are reported, respectively, the parameters, the forecasts and the decision and logical variables used in the mathematical formulation.

\section{SySTEM MODELING AND CONSTRAINTS}

For the sake of brevity, in this section we will often refer to [8], [9] from where many inequalities can be taken.

\section{A. Generator operating constraints}

The thermal power is generated by the power plant using CHP, grate boiler or oil boiler. In our formulation the $i$-th boiler is modeled denoting by $P_{i}(k)$ the power it generates at time $k$, by $T_{i}(k)$ the temperature of the water produced at time $k$ and by $\delta_{i}(k)$ its state, i.e. if the boiler $i$ is ON $\delta_{i}(k)=1$ or zero otherwise. The constraints on the minimum up/down time, ramp up/down power limits, shut down and start up, and to introduce the related costs at the right times can be found in [8], [9]. The generation limits of each boiler can be found in [9]. The fuel consumption cost for boiler unit is traditionally assumed to be a quadratic function of the generated power of the form:

$$
C(P)=a_{1} P^{2}+a_{2} P+a_{3}
$$

where $a_{1}, a_{2}$ and $a_{3}$ are the constant parameters of the generated power.
TABLE I: Parameters

\begin{tabular}{|c|c|}
\hline Parameters & Description \\
\hline$N_{b}$ & number of boilers, \\
\hline$N_{l}, N_{c}, N_{r}$ & $\begin{array}{l}\text { number respectively of critical, curtailable, } \\
\text { and reschedulable power loads }\end{array}$ \\
\hline$C(P)$ & fuel consumption cost curve of a boiler \\
\hline$a_{1}, a_{2}, a_{3}$ & cost coefficients of $C(P)\left[€ /(\mathrm{MWh})^{2}, € / \mathrm{MWh}, €\right]$ \\
\hline$O M$ & operating and maintenance cost of a boiler $[€ / \mathrm{h}]$ \\
\hline$O M^{s}$ & $\begin{array}{l}\text { operating and maintenance cost of the power } \\
\text { exchanged with the storage unit }[€ / \mathrm{h}]\end{array}$ \\
\hline$T_{h}$ & horizon of the optimization problem \\
\hline$X_{\min }^{s}, X_{\max }^{s}$ & $\begin{array}{l}\text { minimum, maximum energy level of the storage } \\
\text { unit [MWh] }\end{array}$ \\
\hline$M^{s}$ & storage power limit [MW] \\
\hline$P_{\min }, P_{\max }$ & minimum, maximum power level of a boiler $[\mathrm{MW}]$ \\
\hline$T_{\min }, T_{\max }$ & $\begin{array}{l}\text { minimum, maximum output water temperature } \\
\text { of a boiler }\left[{ }^{\circ} \mathrm{C}\right]\end{array}$ \\
\hline$\eta^{c}, \eta^{d}$ & storage charging and discharging efficiencies \\
\hline$y_{\min }, y_{\max }$ & $\begin{array}{l}\text { minimum, maximum allowed curtailment } \\
\text { of a curtaible load }\end{array}$ \\
\hline$D^{c}$ & power level required from a curtailable load [MW] \\
\hline$E_{l}$ & total energy required by a reschedulable load [MW] \\
\hline$\left[I T_{l}-F T_{l}\right]$ & time interval set for a reschedulable load $[\mathrm{h}]$ \\
\hline $\bar{P}_{l}^{r, \max }$, & minimum, maximum power levels of \\
\hline$P_{l}^{\prime}$ & a reschedulable load [MW] \\
\hline $\begin{array}{c}\rho_{c} \\
\gamma\end{array}$ & $\begin{array}{l}\text { penalty weight on curtailments } \\
\text { penalty weight on water flow temperature }\end{array}$ \\
\hline$M$ & $\begin{array}{l}\text { mass of the water inside the thermal energy } \\
\text { storage }[\mathrm{kg}]\end{array}$ \\
\hline$c_{p}$ & specific heat $[\mathrm{J} / \mathrm{kgK}]$ \\
\hline$U_{\text {tank }}$ & heat transfer coefficient $\left[\mathrm{W} / \mathrm{m}^{2} \mathrm{~K}\right]$ \\
\hline$A_{\text {tot }}$ & total area of the water storage $\left[\mathrm{m}^{2}\right]$ \\
\hline$q^{+}, q^{-}$ & costs on recourse actions [€/MWh] \\
\hline
\end{tabular}

TABLE II: Forecasts

\begin{tabular}{|c|l|}
\hline Forecasts & Description \\
\hline$P_{\mathrm{RES}}$ & sum of power production from RES [MW] \\
$D$ & power level required from the critical load [MW] \\
$T_{\mathrm{env}}$ & environment temperature $\left[{ }^{\circ} \mathrm{C}\right]$ \\
\hline
\end{tabular}

TABLE III: Decision and logical variables

\begin{tabular}{|c|l|}
\hline Variables & Description \\
\hline$\delta$ & off(0)/on(1) state of a boiler \\
$y_{l}^{r}$ & off(0)/on(1) state of a reschedulable load \\
$P$ & power level of a boiler [MW] \\
$T$ & outlet water temperature of a boiler $\left[{ }^{\circ} \mathrm{C}\right]$ \\
$\delta^{s}$ & discharging $(0) /$ charging $(1)$ mode of the storage unit \\
$P^{s}$ & power exchanged (positive for charging) with the \\
& storage unit [MW] \\
$X^{s}$ & stored energy level [MWh] \\
$\mathrm{SU}, \mathrm{SD}$ & start-up, shut-down costs of a boiler [€] \\
$y^{c}$ & curtailed percentage on the controllable load \\
$y^{r}$ & on/off status of a reschedulable load \\
$p^{r}$ & power consumption of the reschedulable load [MW] \\
$y^{+}, y^{-}$ & recourse variables associated to the loads [MW] \\
\hline
\end{tabular}

\section{B. Thermal Energy Storage modeling}

The thermal storage is described by

$$
\begin{aligned}
X^{s}(k+1)=X^{s} & (k)+\eta P^{s}(k)-U_{\text {tank }} A_{\text {tot }} \\
& \times\left(T_{\text {mean }}(k)-T_{\text {env }}(k)\right),
\end{aligned}
$$

where $T_{\text {mean }}$ represents the average temperature of the water inside the tank,

$$
\eta= \begin{cases}\eta^{c}, & \text { if } P(k)>0 \text { (charging mode) } \\ 1 / \eta^{d}, & \text { otherwise (discharging mode) }\end{cases}
$$


and $0<\eta^{c}, \eta^{d}<1$. Since the energy stored is evaluated as

$$
X^{s}(k)=M c_{p}\left(T_{\text {mean }}(k)-T_{\text {ref }}(k)\right)
$$

where $T_{\text {ref }}(k)$ is the reference temperature at time $k$, we obtain $T_{\text {mean }}(k)$ from the previous equation and thus

$$
\begin{aligned}
X^{s}(k+1)=X^{s} & (k)+\eta P^{s}(k)-U_{\mathrm{tank}} A_{\mathrm{tot}} \\
& \times\left(T_{\mathrm{ref}}(k)+\frac{X^{s}(k)}{M c_{p}}-T_{\mathrm{env}}(k)\right) .
\end{aligned}
$$

The reference temperature $T_{\text {ref }}(k)$ can be chosen equal to the temperature of the return water from the network. The charging and discharging efficiencies are taken into account by using the standard approach described in [24] which employs an auxiliary variable $z^{s}(k)$ and some related inequalities so that the model becomes:

$$
\begin{aligned}
& X^{s}(k+1)=X^{s}(k)+\left(\eta^{c}-1 / \eta^{d}\right) z^{s}(k)+1 / \eta^{d} P^{s}(k) \\
&-U_{\text {tank }} A_{\text {tot }}\left(T_{\text {ref }}(k)+\frac{X^{s}(k)}{M c_{p}}-T_{\text {env }}(k)\right), \\
& \text { s.t. } E_{1}^{s} \delta^{s}(k)+E_{2}^{s} z^{s}(k) \leq E_{3}^{s} P^{s}(k)+E_{4}^{s}
\end{aligned}
$$

see [9] for indications. The inequality

$$
X_{\min }^{s} \leq X^{s}(k) \leq X_{\max }^{s} .
$$

is customary when modeling storage devices. Our approach is sufficiently flexible to accomodate other operating constraints. For example, following the requests of plant operators, we added the following inequality to ensure that, in the case of a failure in the power plant, the energy level available in the storage system is sufficient to satisfy the power demand for one hour, i.e.

$$
X^{s}(k) \geq D(k)
$$

\section{Load demand}

The main objective of a control strategy for a dispatch problem is to guarantee a perfect balance between loads demand and power generation. Loads can be classified as:

- critical loads, i.e. demand related to essential processes that must always be met;

- controllable loads, i.e. loads that can be shifted or shed in supply constraints or emergency situations.

Concerning controllable loads, they can be classified by priority and type as curtailable loads, that can be reduced or shed, if necessary, and reschedulable loads having the characteristic of being able to be allocated across a range of time.

1) Curtailable Loads: We model this kind of loads introducing a continuous variable $y_{h}^{c}(k)$ representing the curtailed power percentage on the controllable load $h$ at time $k$. Hence the constraints for $h=1, \ldots, N_{c}$ are

$$
\begin{gathered}
0 \leq y_{h}^{c}(k) \leq 1 \\
y_{\min }^{c} \leq \sum_{k=1}^{T_{h}} y_{h}^{c}(k) \leq y_{\max }^{c}
\end{gathered}
$$

Equation (9b) constraints the minimum and maximum allowed curtailment of the controllable load $h$-th over the optimization horizon.

2) Reschedulable Loads: Reschedulable loads have the characteristic of being able to be allocated across a range of time. We consider reschedulable loads with fixed power and consecutiveness of load status for $U_{l}$ stages. We indicate with $p_{l}^{r}(k)$ the power consumption of the load $l$ at time $k$ and with the binary variable $y_{l}^{r}(k)$ the on/off status of the $l$-th load in stage $k$. In the next equations, we show the modelling for each of these loads $l$, with $l=1, \ldots, N_{r}$ :

$$
\begin{aligned}
& \bar{P}_{l}^{r, \min } y_{l}^{r}(k) \leq p_{l}^{r}(k) \leq \bar{P}_{l}^{r, \max } y_{l}^{r}(k) \\
& \sum_{k=0}^{T_{h}-1} p_{l}^{r}(k)=E_{l}, \\
& y_{l}^{r}(k)-y_{l}^{r}(k-1) \leq y_{l}^{r}\left(\tau^{\text {on }}\right) \\
& y_{l}^{r}(k)=0 \quad F T_{l} \leq t \leq I T_{l}, \\
& \sum_{k=0}^{T_{h}-1} y_{l}^{r}(k)=U_{l},
\end{aligned}
$$

where $\tau^{\text {on }}=k+1, \ldots, k+T_{i}^{\text {on }}-1, U_{l}$ is the number of time step in which the load has to be on, $I T_{l}$ and $F T_{l}$ (with $I T_{l} \leq F T_{l} \leq T_{h}$ ) are respectively the initial time step where the load $l$ could be turned on and the final time where it has to be fully supplied. Equations (10a) and (10b) guarantee that power and energy requirements for the load are met; (10c) requires the load process be not interruptible; (10d) and (10e) ensure that the load is not on outside the time interval set by the user and that the load process duration is within the prediction horizon.

Given the nature of the receding horizon approach, the state of the controllable loads has to be included into the initial conditions for the next optimization problem, that will be solved using this new information.

\section{Power balance}

The balance between energy production and consumption must be reached at each time $k$; hence the following equality constraints must hold

$$
\begin{gathered}
\sum_{i=1}^{N_{b}} P_{i}(k)-P^{s}(k)+P_{\mathrm{RES}}(k)=\sum_{j=1}^{N_{l}} D_{j}(k) \\
+\sum_{h=1}^{N_{c}}\left(1-y_{h}^{c}(k)\right) D_{h}^{c}(k)+\sum_{l=1}^{N_{r}} p_{l}^{r}(k) .
\end{gathered}
$$

We can rewrite the power balance equation in a compact form by denoting by $\boldsymbol{u}(k)$ the vector of the decision variables, and by $\boldsymbol{w}(k)$ the vector containing the random variables at time $k$, i.e. RES generation, demand. Hence, at time $k$,

$$
\begin{aligned}
& \boldsymbol{u}(k)= {\left[\boldsymbol{P}^{\prime}(k) \boldsymbol{\delta}^{\prime}(k) \boldsymbol{T}^{\prime}(k) \delta^{s}(k) P^{s}(k) X^{s}(k)\right.} \\
&\left.\boldsymbol{y}^{c^{\prime}}(k) \boldsymbol{p}^{r^{\prime}}(k)\right]^{\prime} \in \mathbb{R}^{2 N_{b}+N_{c}+N_{r}+2} \times\{0,1\}^{N_{b}+1}, \\
& \boldsymbol{w}(k)=\left[P_{\mathrm{RES}}(k) \boldsymbol{D}^{\prime}(k) \boldsymbol{D}^{c^{\prime}}(k)\right]^{\prime} \in \mathbb{R}^{1+N_{l}+N_{c}},
\end{aligned}
$$




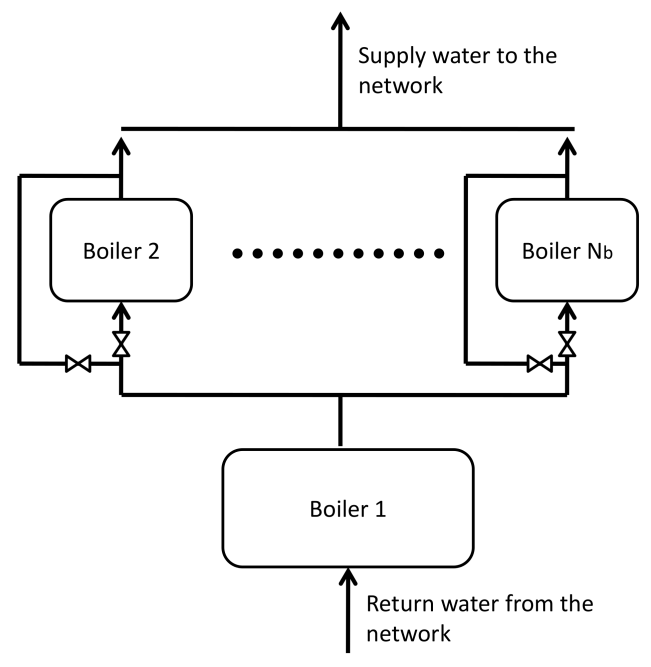

Fig. 1: Layout of the Power Plant

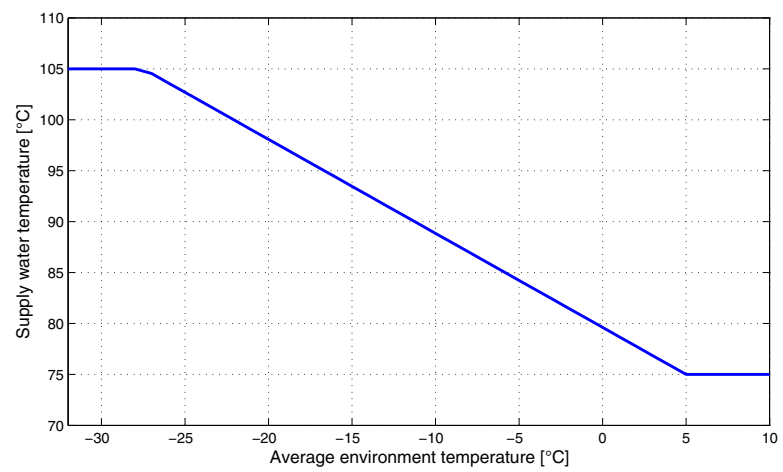

Fig. 2: Supply water temperature in function of the environment temperature

where variables were grouped for compactness, $\boldsymbol{P}(k) \in$ $\mathbb{R}^{N_{b}}, \boldsymbol{\delta}(k) \in\{0,1\}^{N_{b}}, \boldsymbol{T}(k) \in \mathbb{R}^{N_{b}}, \boldsymbol{\beta} \in \mathbb{R}^{N_{c}}, \boldsymbol{y}^{c}(k) \in$ $\mathbb{R}^{N_{c}}, \boldsymbol{p}^{r}(k) \in \mathbb{R}^{N_{r}}, \boldsymbol{D}(k) \in \mathbb{R}^{N_{l}}, \boldsymbol{D}^{c}(k) \in \mathbb{R}^{N_{c}}$.

The power balance can be written as:

$$
\boldsymbol{F}(k) \boldsymbol{u}(k)+\boldsymbol{f} \boldsymbol{w}(k)=0
$$

where $\boldsymbol{F}(k)=\left[\begin{array}{llllllll}\mathbf{1}^{\prime} & \mathbf{0}^{\prime} & \mathbf{0}^{\prime} & 0 & -1 & 0 & \boldsymbol{D}^{c}(k)^{\prime} & -\mathbf{1}^{\prime}\end{array}\right], \boldsymbol{f}=$ $\left[\begin{array}{lll}1 & -\mathbf{1}^{\prime}-\mathbf{1}^{\prime}\end{array}\right]$ with compatible dimensions.

As previously mentioned, a microgrid with CHP includes the ability of supplying both thermal and electric loads; hence the model can be easily generalized considering two balance between energy production and consumption at each time $k$ respectively for the electric and heating components.

\section{E. Power plant layout constraints}

Figure 1 reports the typical layout configuration comprising a main energy source and some auxiliary boilers used to satisfy demand peaks.

The supply temperature is a function of the average daily temperature according to the empirical relationship shown in Figure 2.
The supply water $T_{\text {supply }}(k)$ can either be directly provided by boiler 1 or, in case it exceed boiler 1 maximum output water temperature, it has to be produced by at least one of the auxiliary downstream boilers. This can be expressed as

$$
\text { if } T_{\text {supply }}(k)>T_{1, \max } \text { then } \sum_{i=2}^{N_{b}} \delta_{i}(k) \geq 1 .
$$

The temperature of the outlet water flow coming from each boiler has to respect the following constraints

$$
\begin{aligned}
& T_{1}(k) \geq T_{\text {supply }}(k)\left(1-\sum_{i=2}^{N_{b}} \delta_{i}(k)\right), \\
& T_{i}(k) \geq T_{\text {supply }}(k) \delta_{i}(k) \quad i=2, \ldots, N_{b} .
\end{aligned}
$$

In addition we consider the constraints related to the power plant configuration of the power plant. In particular we consider the layout shown in Figure 1, which is a standard layout for DHNs. In Figure 1 we can see that the boiler 1 is connected in series to the other boilers, whereas the boilers 2,3 and 4 are connected in parallel. This means that when one of the boilers 2, 3 and 4 are turned $\mathrm{ON}$, the outlet water flow rate coming from the boiler 1 becomes the inlet water flow rate to the auxiliary boilers. In this configurations, the boiler 1 is used at maximum of its potentiality.

This situation can be described using the tables IV.

TABLE IV: Constraints related to the auxiliary boilers status

\begin{tabular}{|c|c|}
\hline Auxiliary boiler ON & Auxiliary boiler OFF \\
\hline$T_{1}=T_{1, \max }$ & $T_{1, \min } \leq T_{1} \leq T_{1, \max }$ \\
\hline
\end{tabular}

The constraints related to the layout set-up can be modelled using the set of inequalities

$$
T_{1}(k) \geq T_{1, \max } \delta_{i}(k) \quad i=2, \ldots, N_{b} .
$$

\section{PROBlem DESCRIPTION}

In this section the stochastic MPC problem for optimal DHN operation management is formulated as a MILP problem. In the next sub-sections, we define the cost function and then pose the stochastic MPC problem after defining the cost function.

\section{A. Cost function}

District heating power plant running costs are modeled as follows:

$$
\begin{gathered}
J_{f s}\left(\boldsymbol{u}^{T_{h}}\right)=\sum_{k=0}^{T_{h}-1} \sum_{i=1}^{N_{b}}\left[C_{i}\left(P_{i}(k)\right)+O M_{i} \delta_{i}(k)+S U_{i}(k)+\right. \\
\left.S D_{i}(k)\right]+O M^{s}\left[2 z^{s}(k)-P^{s}(k)\right]+ \\
\gamma T_{i}(k)+\rho_{c} \sum_{h=1}^{N_{c}} y_{h}^{c}(k) D_{h}^{c}(k)
\end{gathered}
$$

where $\gamma T_{i}(k)$ and $\rho_{c} \sum_{h=1}^{N_{c}} y_{h}^{c}(k) D_{h}^{c}(k$ represent, respectively, the penalty on the temperature of the water and the penalty on users' discomfort due to curtailments, and $\boldsymbol{u}^{T_{h}}=$ 
$\left[\boldsymbol{u}(0), \ldots, \boldsymbol{u}\left(T_{h}-1\right)\right]$ is the optimal plan over the prediction horizon. The cost term $\gamma T_{i}(k)$ penalizes two inefficiency sources:

- the energy losses due to a higher water temperature, flowing through the pipes;

- the inefficiency of the boilers, typically lower efficiencies are obtained with higher temperature of the outlet water flow.

The cost function (17) includes also generating and maintenance costs of boilers and TES, as well as shut-down and start-up costs of boilers.

\section{B. Stochastic MPC problem}

The uncertainty during the sampling period $k$ is expressed as $\boldsymbol{w}(k)=\overline{\boldsymbol{w}}(k)+\tilde{\boldsymbol{w}}(k)$, where $\overline{\boldsymbol{w}}(k)$ is the forecast value and $\tilde{\boldsymbol{w}}(k)$ represents the forecast error at time $k$. The forecast values can be computed by using several approaches, such as regression analysis, machine learning techniques, persistent forecasts.

In order to take uncertainty into account, the stochastic nature of renewables and demand is incorporated in the MPC problem. We then propose the use of MPC in combination with stochastic programming with recourse, also known as two-stage stochastic programming [25]. Given the problem under consideration, two-stage stochastic models are a reasonable approach to account for stochasticity into the decision-making process mainly because they are computationally more affordable than multi-stage stochastic programs and allow the use the available recourse actions, which would not be possible if single-stage programs are adopted (e.g., chance-constrained). In two-stage stochastic programs, the decision variables are divided into two groups: the first-stage variables, which have to be decided before the actual realization of the uncertain parameters becomes available, and the second stage or recourse variables, which can be decided once the random events occur. These recourse variables are also interpreted as correction actions as they are used to compensate any infeasibility from the first-stage decisions; thus, violations are accepted, but their costs affect the choice of the first stage variables. Recourse variables represent then the needed amount of power to be compensated for in order to keep the power balance at any point in time. The objective is to choose the first-stage variables in order to minimize the sum of first-stage costs and the expected value of the random second stage or recourse costs.

The two-stage problem for DHN optimal operation management is stated as follows

$$
\min _{\boldsymbol{u}^{T_{h}}} J_{f s}\left(\boldsymbol{u}^{T_{h}}\right)+\mathcal{Q}\left(\boldsymbol{u}^{T_{h}}\right)
$$

Storage dynamic and physical constraints

(6), (7) and (8)

Boilers operating constraints [9]

Controllable loads constraints (9) and (10)

Layout operating mode and heating curve

(14), (15) and (16). where $J_{f s}$ becomes the first-stage cost function and $\mathcal{Q}\left(\boldsymbol{u}^{T_{h}}\right)=E_{\boldsymbol{w}^{T_{h}}}\left[Q\left(\boldsymbol{u}^{T_{h}}, \boldsymbol{w}^{T_{h}}\right)\right]$ is the expected recourse, with $\boldsymbol{w}^{T_{h}}=\left[\boldsymbol{w}(0), \ldots, \boldsymbol{w}\left(T_{h}-1\right)\right]$ being the vector of random variables. The second-stage function, $Q\left(\boldsymbol{u}^{T_{h}}, \boldsymbol{w}^{T_{h}}\right)$ is defined as follows:

$$
\begin{aligned}
& Q\left(\boldsymbol{u}^{T_{h}}, \boldsymbol{w}^{T_{h}}\right)=\min \left(\boldsymbol{q}^{+} \boldsymbol{y}^{+}+\boldsymbol{q}^{-} \boldsymbol{y}^{-}\right) \\
& \text {s.to } \\
& \boldsymbol{y}^{+} \geq \tilde{\boldsymbol{F}} \boldsymbol{u}^{T_{h}}+\tilde{\boldsymbol{f}} \boldsymbol{w}^{T_{h}} \\
& \boldsymbol{y}^{-} \geq-\left(\tilde{\boldsymbol{F}} \boldsymbol{u}^{T_{h}}+\tilde{\boldsymbol{f}} \boldsymbol{w}^{T_{h}}\right) \\
& \boldsymbol{y}^{+}, \boldsymbol{y}^{-} \geq 0
\end{aligned}
$$

where $\boldsymbol{y}^{+}$and $\boldsymbol{y}^{-}$are recourse variables representing thermal power surplus and shortage respectively, $\boldsymbol{q}^{+}$and $\boldsymbol{q}^{-}$are penalty costs related to power surplus and shortage respectively, $\tilde{\boldsymbol{F}}=\operatorname{diag}\left(\boldsymbol{F}(0), \ldots, \boldsymbol{F}\left(\mathrm{T}^{\mathrm{h}}-1\right)\right)$ and $\tilde{\boldsymbol{f}}=\boldsymbol{f} \otimes I_{T \times T}$, with $\otimes$ denoting the Kronecker product. We remark that the power balance constraint is a second-stage constraint since it involves random variables.

To derive a tractable approximation of the stochastic MPC problem (18), we approximate the actual probability distribution of the uncertainty as a discrete distribution with a certain number of outcomes, which are used for generating a finite number of paths over the prediction horizon, i.e. scenarios. In particular, we apply a known sampling technique to obtain an approximate value of the second-stage function, the Sample Average Approximation (SAA) [22], [26]: we consider a finite number of randomly selected scenarios and then solve a deterministic optimization problem.

Suppose that a random sample $\hat{\boldsymbol{w}}_{1}^{T}, \ldots, \hat{\boldsymbol{w}}_{S}^{T}$ of $S$ realizations of the random vector $\boldsymbol{w}^{T}$ is generated. The SAA of the second-stage function is defined as:

$$
\hat{\mathcal{Q}}\left(\boldsymbol{u}^{T_{h}}\right):=\frac{1}{S} \sum_{i=1}^{S} Q\left(\boldsymbol{u}^{T_{h}}, \boldsymbol{w}_{i}^{T_{h}}\right)
$$

where

$$
\begin{aligned}
& Q\left(\boldsymbol{u}^{T_{h}}, \boldsymbol{w}_{i}^{T_{h}}\right)=\min \left(\boldsymbol{q}^{+} \boldsymbol{y}_{i}^{+}+\boldsymbol{q}^{-} \boldsymbol{y}_{i}^{-}\right) \\
& \text {s.to } \\
& \boldsymbol{y}_{i}^{+} \geq \tilde{\boldsymbol{F}} \boldsymbol{u}^{T_{h}}+\tilde{\boldsymbol{f}} \hat{\boldsymbol{w}}_{i}^{T_{h}} \\
& \boldsymbol{y}_{i}^{-} \geq-\left(\tilde{\boldsymbol{F}} \boldsymbol{u}^{T_{h}}+\tilde{\boldsymbol{f}} \hat{\boldsymbol{w}}_{i}^{T_{h}}\right) \\
& \boldsymbol{y}_{i}^{+}, \boldsymbol{y}_{i}^{-} \geq 0
\end{aligned}
$$

Since the random realizations $\hat{\boldsymbol{w}}_{i}^{T}$, for $i=1, \ldots, S$, have the same probability distribution, it follows that $\hat{\mathcal{Q}}\left(\boldsymbol{u}^{T_{h}}\right)$ is an unbiased estimator of $\mathcal{Q}\left(\boldsymbol{u}^{T_{h}}\right)$, for any $\boldsymbol{u}^{T_{h}}$. We used Latin Hypercube sampling (LHS) to construct the SAA function, which allows to compute an unbiased estimator with considerably less variance than the one obtained from Monte Carlo sampling techniques (see [22] and references therein for details on the LHS method).

Following the procedure described in [22], we can identify the sample size required to obtain approximate solutions of reasonable quality. We calculate estimates of upper and lower bounds on the optimal value of the original stochastic problem (18), and of the optimality gap, by solving many 
SAA instances for different sample sizes and using Latin Hypercube sampling (LHS) as sampling technique. LHS has been proved to compute an unbiased estimator with considerably less variance than the one obtained from Monte Carlo sampling technique (see [22] and references therein for details on the LHS method). Therefore, LHS produces improvements in both the optimality gap and the variance of the upper and lower bound estimates.

In closing, the stochastic MPC optimization problem to be solved is:

$$
\min _{\boldsymbol{u}^{T_{h}}} J_{f s}\left(\boldsymbol{u}^{T_{h}}\right)+\frac{1}{S} \sum_{i=1}^{S}\left(\boldsymbol{q}^{+} \boldsymbol{y}^{+}+\boldsymbol{q}^{-} \boldsymbol{y}^{-}\right)
$$

s.t.

Storage dynamic and physical constraints

(6), (7) and (8)

Boilers operating constraints [9]

Power balance constraints (20c), (20d) and (20e)

Controllable loads constraints (9) and (10)

Layout operating mode and heating curve

(14), (15) and (16).

To further compensate for the difference between prediction and actual states, the described optimization problem is embedded in an MPC framework. This means that, at the current point in time, an optimal plan is formulated but only the first sample of the input sequence is implemented, and subsequently the horizon $T_{h}$ is shifted. At the next sampling time, the new state of the system is measured, and a new optimization problem is solved using this new information.

\section{Simulation EVAluation}

The proposed control strategy has been evaluated on a real case study, the DHN of Ylivieska, a small town in the north-west of Finland. In this section we describe the DHN established in Ylivieska and currently in use, and we evaluate the performance of the stochastic MPC controller described in the previous sections.

\section{A. Descritpion of the case study}

The DHN in Ylivieska has $53 \mathrm{~km}$ of pipes delivering hot water to almost 600 customers. The district heating power plant consists in: a CHP, a grate boiler, two oil boilers (respectively boiler 1 , boiler 2 , boiler 3 and 4 in figure 1) and a thermal energy storage. The electric power generated by the CHP is not considered since it is used in practice for specific, localized district operations. There are not flexible loads and no solar power plants are currently utilized, i.e. $P_{\text {RES }}=0$.

The generation costs of each boiler can be represented by the linear term $C(P)=a_{2} P$ in (1).

Table $\mathrm{V}$ reports the main parameters of each boiler in the power plant. The additional costs related to the recourse actions are $q^{+}=q^{-}=50[€ / \mathrm{MWh}]$.

The power generation costs are discussed in Appendix I.
TABLE V: Table of the boilers parameters

\begin{tabular}{|c|c|c|c|c|c|c|}
\hline Unit & $\begin{array}{c}P_{\min } \\
{[\mathrm{MW}]}\end{array}$ & $\begin{array}{c}P_{\max } \\
{[\mathrm{MW}]}\end{array}$ & $\begin{array}{c}T_{\min } \\
{ }^{\circ} \mathrm{C}\end{array}$ & $\begin{array}{c}T_{\max } \\
{ }^{\circ} \mathrm{C}\end{array}$ & $\begin{array}{c}a_{2} \\
{[€ / \mathrm{MWh}]}\end{array}$ & $\begin{array}{c}R_{\max } \\
{[\mathrm{MW} / \mathrm{h}]}\end{array}$ \\
\hline CHP & 5 & 20 & 80 & 90 & 14.4 & 3 \\
\hline Grate & 2 & 12 & 105 & 115 & 23.8 & 6 \\
\hline K3 & 1 & 12 & 112 & 112 & 71.1 & 12 \\
\hline K2 & 1 & 6 & 120 & 120 & 100.7 & 6 \\
\hline
\end{tabular}

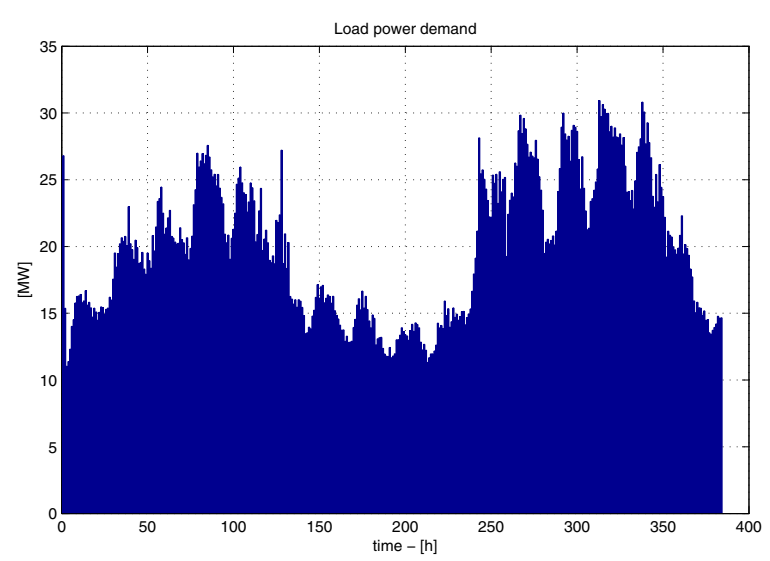

Fig. 3: Aggregated demand over the simulation horizon

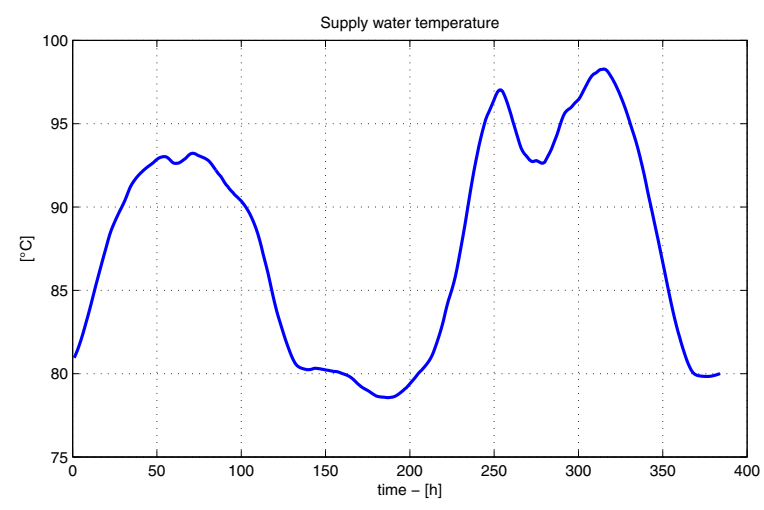

Fig. 4: Supply water temperature

In order to model uncertainty and generate scenarios, baseline power demand forecasts have been provided by the Finnish DHN operator and errors have been computed based on historical data.

\section{B. Simulation results}

We consider a sampling period of $1 \mathrm{~h}$, an optimization horizon of $12 \mathrm{~h}$ and a simulation horizon of 15 days.

The power demand 3 and the environment temperature used for the simulation study are real measurements retrieved from the Ylivieska Database system (from 9 of January 2015 to 23 of January 2015).

The supply water temperature in 4 has been computed using the relationship in Figure 2 and measurements of the environment temperature.

Utilizing the data from Ylivieska Database system, we performed the testing procedure described in [26] to determine lower and upper bound estimates on the optimal value of 

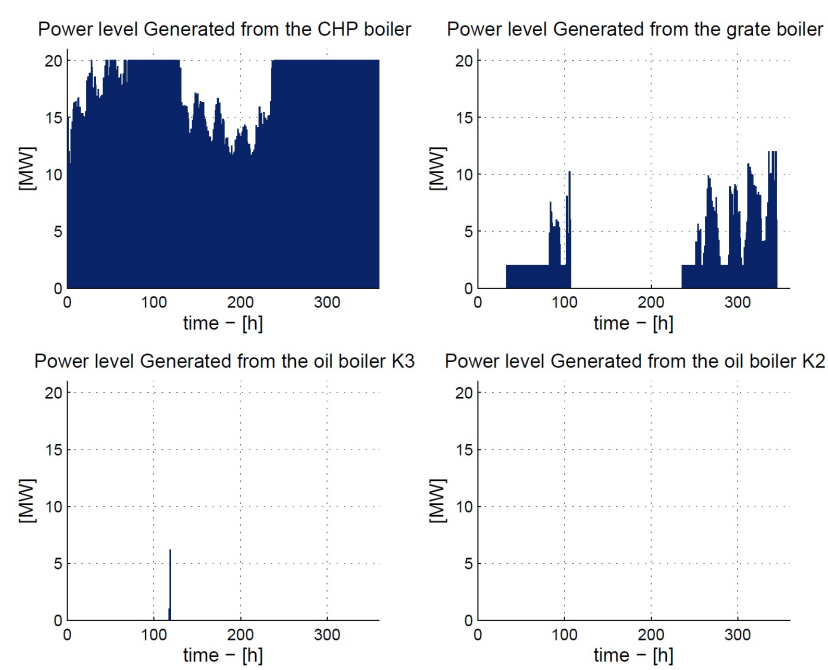

Fig. 5: Boilers' power level over the simulation horizon
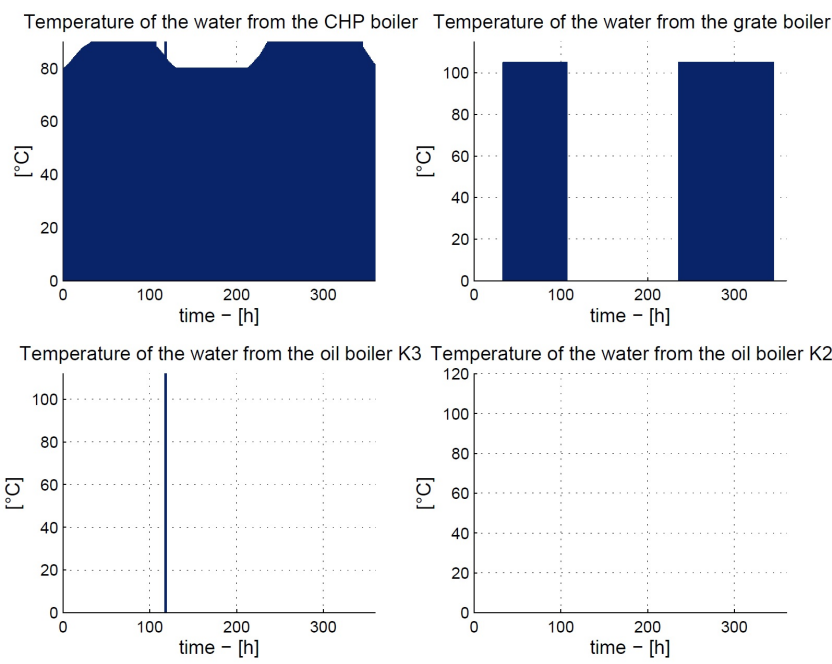

Fig. 6: Boilers' outlet water temperature over the simulation horizon

the original stochastic problem and identify the appropriate number of scenarios. We solved 50 instances of the SAA problem (21) with a sample size, $N_{s}$, varying from 10 to 1000 and compared the obtained bounds. A sample size of $N_{s}=100$ scenarios is required to obtain solutions of reasonable quality (with a variance of the upper and lower bound estimates of 0.01).

Considering an initial storage energy level of $X^{s}(0)=$ $30 \mathrm{MW}$ and $\delta_{1}(0)=1$, the stochastic MPC controller has been applied. Figures 5 and 6 show respectively the power level and the temperature of the outlet water flow rate of each boiler.

We can notice that CHP is mainly used to meet the power demand, since it is the cheapest boiler. When $T_{\text {supply }}(k)>$ $T_{1, \max }$ the Grate boiler is mainly employed. The thermal energy storage can reduce the usage of the most expensive oil boiler. The oil boilers is only used when the peak demand

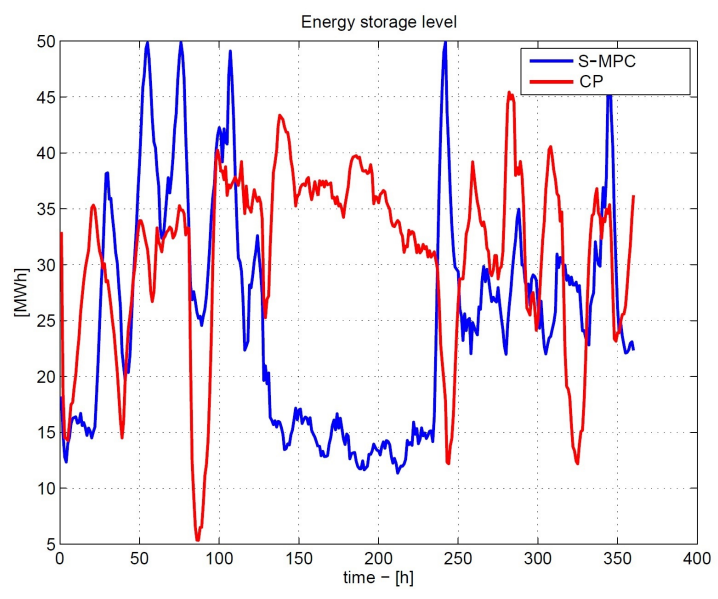

Fig. 7: Storage energy profiles: comparison between S-MPC and $\mathrm{CP}$

lasts shortly so that it is not convenient to turn on the Grate boiler, whose minimum up time is $8 \mathrm{~h}$.

\section{Comparison analysis}

We compare the following strategies: i) Stochastic MPC (S-MPC); ii) Deterministic MPC (D-MPC), which is an MPC controller that uses only forecasts of random variables and does not take forecast errors into account (thus it solves (21) without the second-stage function and with the power balance constraints (11)); iii) Benchmark MPC (B-MPC), which is an ideal MPC controller that uses the actual values of the random variables; $i v$ ) Current Practice (CP), based on power, energy and costs data from Ylivieska Database relating to the same time period (9-23 January 2015).

The total running costs are reported in table VI. These costs include the costs of corrective actions required for compensating for the actual power imbalances; for example, in case of power shortage, to prevent users' discomfort, this deficit is to be either discharged from the energy storage, if possible, or generated by a running boiler, leading to additional generation costs. The stochastic MPC controller yields $98 \%$ reduction of imbalances compared to the deterministic MPC (15MWh against 1225MWh over 15 days). Further, costs obtained by the S-MPC controller are the closest to the benchmark.

Figure 7 shows the storage energy profiles obtained by applying the S-MPC control scheme and the CP. The S-MPC keeps the energy inside the storage as low as possible when the power demand from the network is low, while it is able to pre-charge the thermal energy storage to properly meet upcoming occurring demand peaks.

All the formulations described above have been implemented using MatLab. GLPK has been employed to solve MILP optimization problems. All computations are performed on an Intel Core i7 CPU, $1.80 \mathrm{GHz}$; computational time for each iteration is less than 0.7 seconds on average. 
TABLE VI: Results evaluation

\begin{tabular}{|c|c|c|c|c|}
\hline Unit & S-MPC [€] & D-MPC $[€]$ & B-MPC [€] & CP [€] \\
\hline CHP boiler & 96015 & 99378 & 92874 & 91378 \\
\hline Grate boiler & 21903 & 21684 & 20162 & 33145 \\
\hline Oil boiler K3 & 579 & 5602 & 0 & 320 \\
\hline Oil boiler K2 & 0 & 0 & 0 & 0 \\
\hline total costs & 123820 & 126660 & 118540 & 124990 \\
\hline
\end{tabular}

TABLE VII: Power generation costs.

\begin{tabular}{cc}
\hline Fuel & $\begin{array}{c}\text { Cost } \\
{[€ / \mathrm{MWh}]}\end{array}$ \\
\hline CHP & 14.4 \\
Grate (K1) & 23.8 \\
Oil boiler (K2) & 100.7 \\
Oil boiler (K3) & 71.1 \\
\hline
\end{tabular}

\section{CONCLUSION}

In this paper, we have designed a stochastic predictive control strategy for optimal operation management of district heating power plants. The problem is stated as a mixed integer linear model, since to properly represent system dynamics both continuous and logical variables are needed. A stochastic formulation of this model, incorporated into a model predictive control framework, is proposed to account for renewable and demand power uncertainty effects. The stochastic controller is evaluated via simulation on an existing district heating power plant. Results show that the proposed strategy can considerably reduce power imbalances and outperform the deterministic controller and the current practice in terms of cost savings. Ongoing and future works consider the application of the stochastic controller to the Finnish district heating network, the extension to multienergy carriers and the design of distributed approaches.

\section{APPENDIX I}

\section{ENERGY PRODUCTIONS COSTS}

The fuels used in the power plant are: light and heavy combustion oil, peat bricks (used into the Grate boiler), the wood chips and the granular peat used to feed the steam generator of the CHP.

In table VII the generation costs per unit of thermal power are provided.

\section{REFERENCES}

[1] M. Frling, C. Reidhav, J. Olof Dalenbck, and S. Werner, "Is there a role for district heating in future cities with low energy buildings?" in The 11th International Symposium on District Heating and Cooling, 2008.

[2] K. Sartor, S. Quoilin, and P. Dewallef, "Simulation and optimization of a CHP biomass plant and district heating network," Applied Energy, vol. 130, pp. $474-483,2014$.

[3] M. Ameri and Z. Besharati, "Optimal design and operation of district heating and cooling networks with CCHP systems in a residential complex," Energy and Buildings, vol. 110, pp. 135 - 148, 2016.

[4] Y. Yang, S. Zhang, and Y. Xiao, "Optimal design of distributed energy resource systems coupled with energy distribution networks," Energy, vol. 85, pp. $433-448,2015$.

[5] H. Wang, W. Yin, E. Abdollahi, R. Lahdelma, and W. Jiao, "Modelling and optimization of CHP based district heating system with renewable energy production and energy storage," Applied Energy, vol. 159, pp. $401-421,2015$.

[6] H. Yang, T. Xiong, J. Qiu, D. Qiu, and Z. Y. Dong, "Optimal operation of des/cchp based regional multi-energy prosumer with demand response," Applied Energy, pp. -, 2015, available online on 30 November 2015 - In Press, Corrected Proof.
[7] A. Shabanpour-Haghighi and A. R. Seifi, "Effects of district heating networks on optimal energy flow of multi-carrier systems," Renewable and Sustainable Energy Reviews, vol. 59, pp. 379 - 387, 2016.

[8] A. Parisio, E. Rikos, and L. Glielmo, "A model predictive control approach to microgrid operation optimization," Control Systems Technology, IEEE Transactions on, vol. 22, no. 5, pp. 1813-1827, Sept 2014.

[9] G. Gambino, F. Verrilli, D. Meola, M. Himanka, C. Del Vecchio, and L. Glielmo, "Model predictive control for optimization of combined heat and electric power microgrid," in The 19th edition of world congress, IFAC, 2014.

[10] A. Parisio and L. Glielmo, "Stochastic model predictive control for economic/environmental operation management of microgrids," in Control Conference (ECC), 2013 European, July 2013, pp. 2014-2019.

[11] L. Yang, M. He, V. Vittal, and J. Zhang, "Stochastic optimization based economic dispatch and interruptible load management with distributional forecast of wind farm generation," in Decision and Control (CDC), 2014 IEEE 53rd Annual Conference on, Dec 2014, pp. 199-204.

[12] Z. Yu, L. Jia, M. Murphy-Hoye, A. Pratt, and L. Tong, "Modeling and stochastic control for home energy management," Smart Grid, IEEE Transactions on, vol. 4, no. 4, pp. 2244-2255, Dec 2013.

[13] E. Carpaneto, G. Chicco, P. Mancarella, and A. Russo, "Cogeneration planning under uncertainty. part ii: Decision theory-based assessment of planning alternatives," Applied Energy, vol. 88, no. 4, pp. 1075 1083,2011

[14] S. M. Lai and C. W. Hui, "Integration of trigeneration system and thermal storage under demand uncertainties," Applied Energy, vol. 87, no. 9 , pp. $2868-2880,2010$.

[15] T. Fang and R. Lahdelma, "Optimization of combined heat and power production with heat storage based on sliding time window method," Applied Energy, vol. 162, pp. 723 - 732, 2016.

[16] Y. Kitapbayev, J. Moriarty, and P. Mancarella, "Stochastic control and real options valuation of thermal storage-enabled demand response from flexible district energy systems," Applied Energy, vol. 137, pp. $823-831,2015$

[17] M. R. Benam, S. S. Madani, S. M. Alavi, and M. Ehsan, "Optimal configuration of the chp system using stochastic programming," IEEE Transactions on Power Delivery, vol. 30, no. 3, pp. 1048-1056, June 2015.

[18] N. Good, E. Karangelos, A. Navarro-Espinosa, and P. Mancarella, "Optimization under uncertainty of thermal storage-based flexible demand response with quantification of residential users' discomfort," IEEE Transactions on Smart Grid, vol. 6, no. 5, pp. 2333-2342, Sept 2015.

[19] G. Angrisani, M. Canelli, C. Roselli, and M. Sasso, "Calibration and validation of a thermal energy storage model: Influence on simulation results," Applied Thermal Engineering, vol. 67, no. 12, pp. 190 - 200, 2014.

[20] R. M. Dickinson, C. A. Cruickshank, and S. J. Harrison, "Charge and discharge strategies for a multi-tank thermal energy storage," Applied Energy, vol. 109, no. 0, pp. 366 - 373, 2013.

[21] S. Kim, R. Pasupathy, and S. Henderson, "A guide to sample average approximation," in Handbook of Simulation Optimization, ser. International Series in Operations Research \& Management Science, M. C. Fu, Ed. Springer New York, 2015, vol. 216, pp. 207-243.

[22] J. Linderoth, A. Shapiro, and S. Wright, "The empirical behavior of sampling methods for stochastic programming," Annals of Operations Research, vol. 142, no. 1, pp. 215-241, 2006.

[23] J. R. Birge and F. Louveaux, Introduction to Stochastic Programming. Springer-Verlag New York, 2011.

[24] A. Bemporad and M. Morari, "Control of systems integrating logic, dynamics, and constraints," Automatica, vol. 35, no. 3, pp. 407-427, 1999

[25] P. Kall and S. Wallace, Stochastic programming. John Wiley and Sons Ltd, 1994.

[26] A. Ruszczynski and A. Shapiro, Stochastic Programming, Handbook in Operations Research and Management Science. Elsevier, 2003. 